\title{
APPLICATION OF ANALYTICAL COLOR FLUORESCENCE ELECTRON MICROSCOPY TO BIOMEDICAL FIELD: I. VITAMIN A ESTER IN RAT RETINA
}

\author{
Tohru NAKANO, Toyoshi FUJIMOTO, Hirotami KOIKE* \\ and Kazuo OGAWA \\ Department of Anatomy, Faculty of Medicine, Kyoto University, Sakyo-ku, \\ Kyoto 606 and *Akashi Beam Technology Corp., Hachioji, Tokyo 192
}

Received for publication November 30, 1990

\begin{abstract}
An analytical color fluorescence electron microscope (ACFEM) based on a high-resolution scanning electron microscope has been developed. The ACFEM enables us not only to detect cathodoluminescence (CL), which is a weak luminescence signal under electron beam bombardments, as color images, but also to analyze CL spectra. A cryo-SEM method was introduced to prevent beam effect on biological specimens. In experiments 1 and 2, we observed adult rat retinas under different conditions: hyper- and hypovitaminosis $A$ and light and dark adaptation, which revealed that the distribution of vitamin $A$ ester and its change under these conditions could be detected in situ by the ACFEM. In experiment 3, postnatal development of rat retina was observed under the ACFEM up to 3 weeks after birth. The retinal pigment epithelial cells of new born rats were already functioning as vitamin $A$ storing cells. On the other hand, vitamin $A$ ester in the developing outer segment first appeared on the 13th postnatal day, which suggests a correlation to the development of visual function. These results show that $C L$ analysis by the ACFEM is a simple and effective new method in the field of histo- and cytochemistry.
\end{abstract}

In scanning electron microscopy, several signals can be utilized such as secondary electrons, backscattered electrons, transmitted electrons, Auger's electrons and Xrays. At the same time, cathodoluminescence (CL) is emitted as a light signal in the visible, ultraviolet and infrared regions when substances are bombarded with an electron beam (30). Although the CL phenomenon is essentially similar to fluorescence and phosphorescence elicited by ultraviolet light, it has some intrinsic advantages over them $(6,30):$ 1) CL can be amplified electronically and measured quantitatively, 2) the depth of focus and the resolution in the CL mode is higher than in the conventional fluorescence microscope, 3) some substances exhibit luminescence only under electron irradiation. In the industrial field, the CL technique has been introduced as a practically valuable tool for analyzing wide band-gap material such as diamonds (26). However, in the biomedical field, although many attempts have been made to

Correspondence to: Tohru Nakano, Department of Anatomy, Faculty of Medicine, Kyoto University, Konoe-cho, Yoshida, Sakyo-ku, Kyoto 606, JAPAN.

This study was supported by a Grant-in-Aid for Developmental Scientific Research from the Ministry of Education, Science and Culture, Japanese Government, No. 01870002. 
utilize CL (2-4, 6-10, 17, 29, 32, 33), its applications have been limited, because CL from biological material is a very weak luminescence signal and easily fades under high beam current (beam effect) (10). Nevertheless, the CL analysis technique is an attractive new method even in the biomedical field, because the CL from biomolecules is derived from loosely bound $\pi$-electrons and gives us information about the chemical bond (9), which has not been obtained using conventional analytical electron microscopic technique such as X-ray analysis.

In previous studies $(19,22)$, we have developed an analytical color fluorescence electron microscope (ACFEM) based on a high resolution scanning electron microscope (SEM). The ACFEM enabled us to detect CL from biological specimens as a color image simultaneously with secondary electron (SE) images and also to analyze their CL spectra. Recently, we introduced a new system for spectrometric CL analysis by which the time needed for CL spectrometry was significantly reduced, as was the beam effect on specimens.

In this study, rat retinas under several different conditions were observed under the ACFEM using cryo-SEM technique, and the distribution of vitamin A ester and its dynamic changes were detected in situ, which suggested that the CL analysis by the ACFEM is a simple and effective new method in the field of histo- and cytochemistry.

\section{INSTRUMENTS}

The ACFEM was developed on the basis of a high resolution SEM (ISI-DS130; Akashi Beam Technology Corp., Tokyo) (22) (Fig. 1). This SEM has two stages for specimens, a top stage for ultra-high resolution observation $(3 \mathrm{~nm}$ with a tungsten filament and $2 \mathrm{~nm}$ with a $\mathrm{LaB}_{6}$ filament) and a bottom stage for the observation of large specimens. In this study, the top stage was used to observe CL in color and to analyze CL spectra.

In order to maintain high performance at the low acceleration voltages which should be used to avoid the beam effect in the CL mode, a high-excitation objective lens was introduced. Three ellipsoidal mirrors were arranged at 120 degrees to each other and combined so as to hold a single focus accurately. This set of three combined ellipsoidal mirrors has a small hole in the center for electrons to pass through to the specimens, and is placed below the upper objective lens pole-piece as a collection system for CL photons. Considering the angle distribution of CL emission and solid angle of the mirror, about $90 \%$ of CL photons should be detected. At the other focal points of the three ellipsoidal mirrors, red, green and blue filters were placed. A photomultiplier tube (PMT) was placed next to each filter, and the CL, divided into three components, was amplified by the PMT and integrated to form a single color image through a memory system. For CL spectrometry, a monochrometer (HR-320; Jobin-Yvon, U.S.A.) was placed at a focal point of an ellipsoidal mirror followed by a spectrometric multichannel analyzer (SMA; Princeton Instruments Inc., U.S.A.) and SMA control system. Monochromatic CL images at any wavelength could be obtained by attaching a PMT next to the monochrometer (Fig. 2).

A cryo-holder was adopted to minimize the beam effect, and was kept cool at about $-120^{\circ} \mathrm{C}$ by liquid nitrogen during observation. 




FIG. 1. Outside view of the ACFEM (the SMA system is not included here).

\section{MATERIALS AND METHODS}

In experiments 1 and 2, adult male and female Wistar rats were used. These two experiments were designed to elucidate the detectability of vitamin A compounds in rat retinas by the ACFEM. Experiment 3 was performed to reveal the distribution of vitamin A compounds in developing rat retinas by means of CL.

Experiment 1. hyper- and hypovitaminosis A

Hypervitaminosis A rats were produced by intraperitoneal injection of 5,000 IU/ day of vitamin A palmitate (Chocola A; Eisai Co., Ltd., Tokyo) for a week. Hypovitaminosis A rats were produced by feeding them with vitamin A-free diet (Oriental Koubo Co., Tokyo) for 10 weeks following suckling periods. Both groups were kept under the same condition of light and dark cycle, and the specimen preparations were processed under a normal room light.

Experiment 2. light and dark adaptation

The animals were kept in complete darkness for $6 \mathrm{hr}$, and then the group for the light adaptation was placed $50 \mathrm{~cm}$ below a $200 \mathrm{~W}$ white incandescent light bulb (light intensity about $8 \mathrm{~mW} / \mathrm{cm}^{2}$ ) for $60 \mathrm{~min}$.

Experiment 3. postnatal development of rat retina

Infant rats were sacrificed various days after birth until 3 weeks old. The infant 


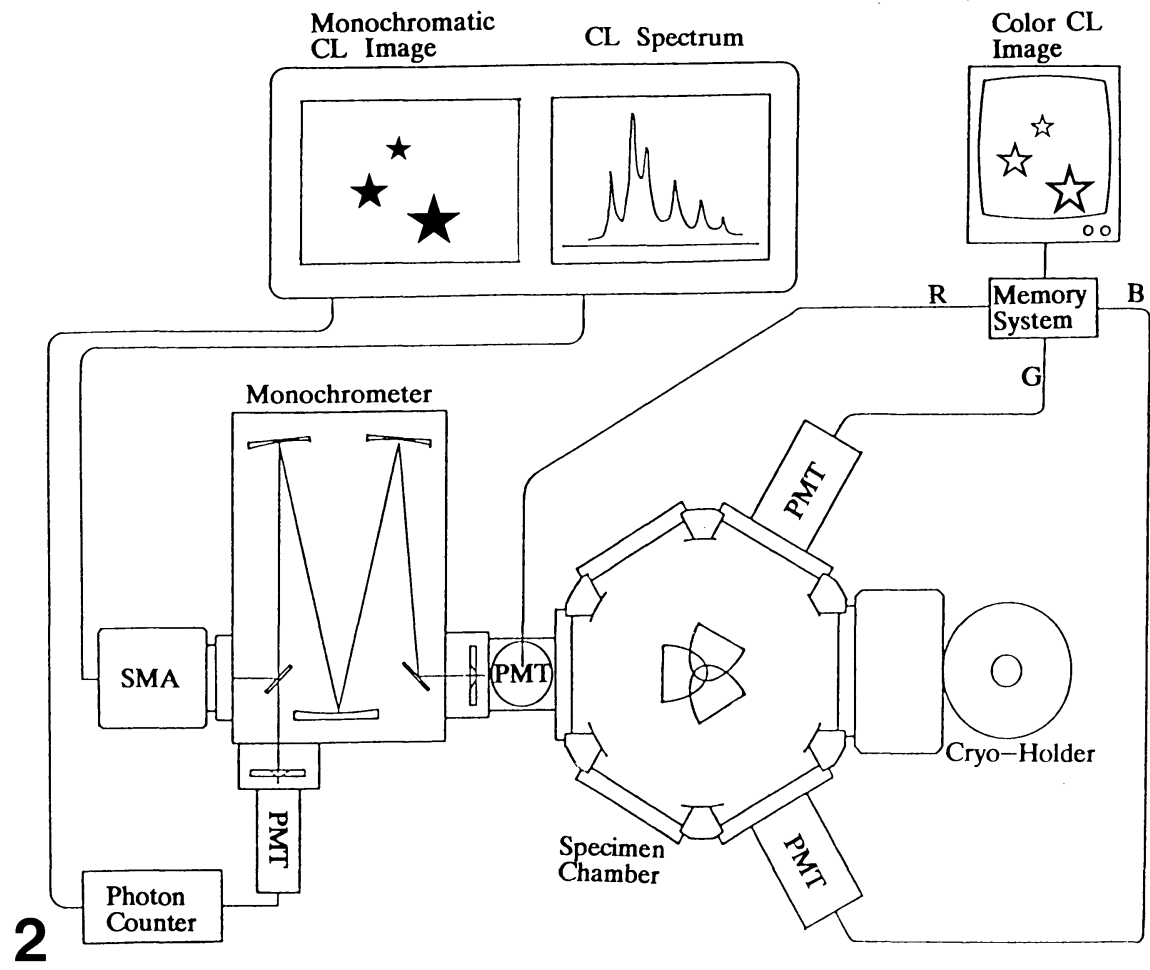

FIg. 2. Schematic diagram of the ACFEM. GL, divided into three components (R, G, B), is reconstructed into a single color image. For CL spectrometry and monochromatic CL imaging, the monochrometer is placed at a focus of an ellipsoidal mirror.

animals were kept with their mothers under the same conditions of light and dark cycle, and the specimen preparations were processed under a normal room light.

All rats, except for those within one week after birth, were fixed by transcardial infusion with $2 \%$ formaldehyde in $0.1 \mathrm{M}$ phosphate buffer, $\mathrm{pH} 7.4$ for $5 \mathrm{~min}$ to prevent retinal detachment. The enucleated eyeballs were cut into small pieces (about $2 \times 4 \mathrm{~mm}$ ) in phosphate buffered saline solution. In the case of animals within one week after birth in experiment 3 , whole eye balls were used after decapitation and enucleation. These specimens were mounted on small aluminum cryo-tips and rapidly frozen in liquid propane precooled by liquid nitrogen. The specimens of dark adapted rats in experiment 2 were processed under dim red light up to this step. The surface of the specimens was then cut by a cryo-ultramicrotome (Ultracut E, FC-4; Reichert, Austria) at $-100^{\circ} \mathrm{C}$ to expose the intracellular structure and to make the surface flat for equal detection of the CL by the three ellipsoidal mirrors. Finally, the specimens were attached to the cryo-holder and observed under the ACFEM. Observations were done under accelerating voltages of $5-9 \mathrm{kV}$, and a thin gold coating was performed inside the column of the electron microscope to observe the SE images. 


\section{RESULTS}

In this study, we used chemical fixation by formaldehyde to preserve the stratum structure of the retina, because otherwise retinal detachment could not be avoided when the eye ball was dissected into small pieces before freezing. In a preliminary experiment, no spectral change in GL was observed between unfixed and chemically fix-
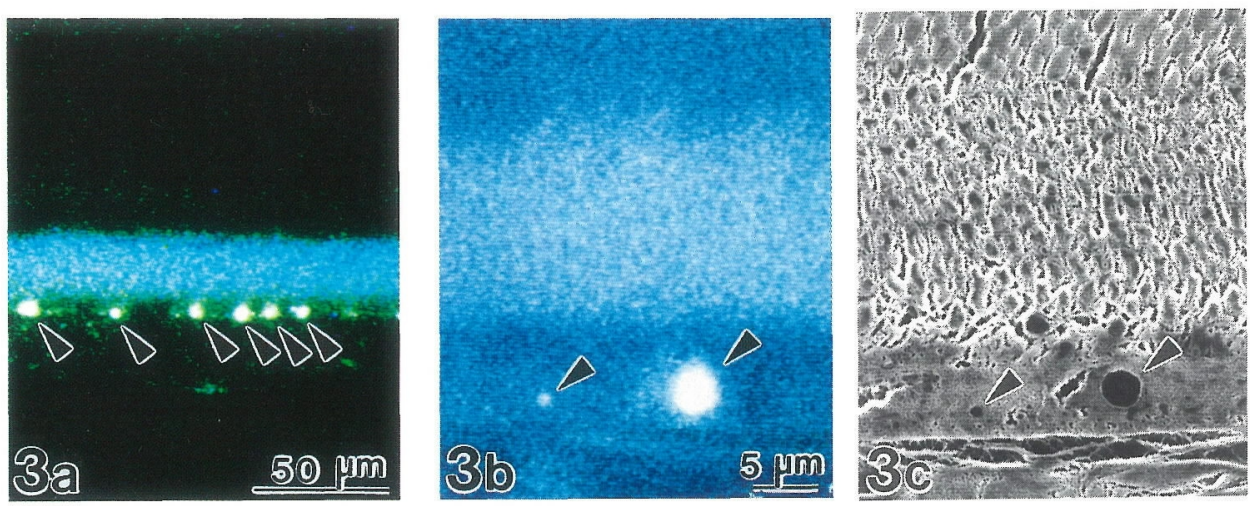

Figs. 3a, b, c. Color CL (a, b) and secondary electron image (c: corresponding to b) of a hypervitaminosis A rat retina. The outer segment (OS) and lipid droplets (arrowheads) of the retinal pigment epithelial cells (RPE) emit strong CL.

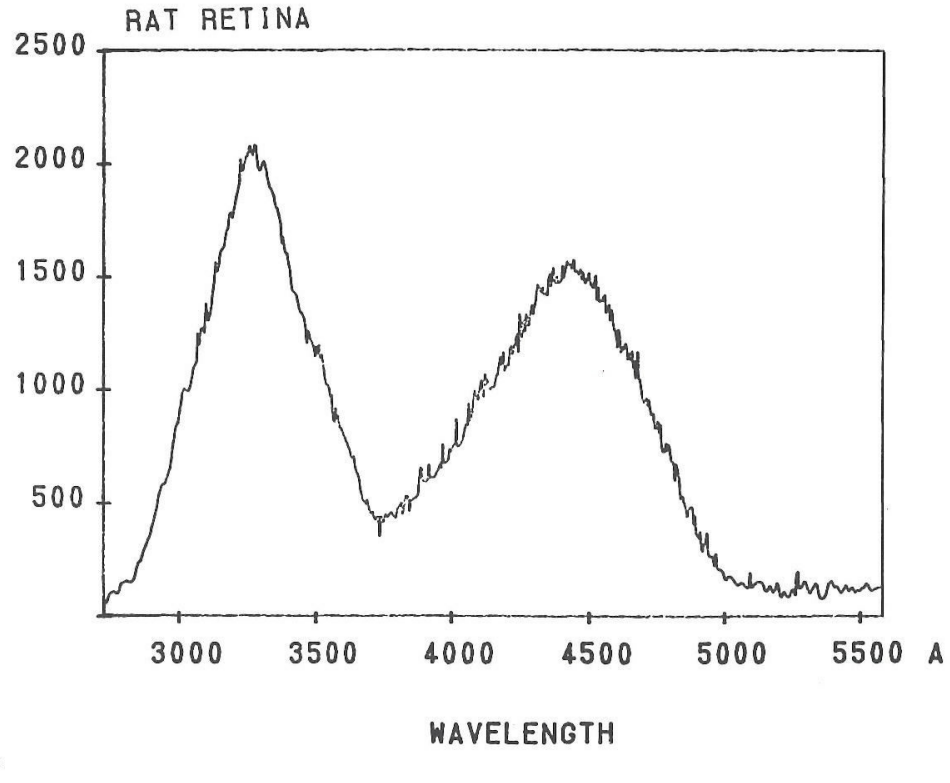

FIG. 4. CL spectrum of hypervitaminosis A rat retina. Two wavelength peaks (325 $\mathrm{nm}, 445 \mathrm{~nm})$ are observed. 



FIGs. 5a, b. Color CL (a) and secondary electron (b) image of a hypovitaminosis A rat retina. The CL intensity is much weaker than that of the hypervitaminosis A rat, and the OS membrane is destroyed (arrowheads). Bars $=10 \mu \mathrm{m}$.
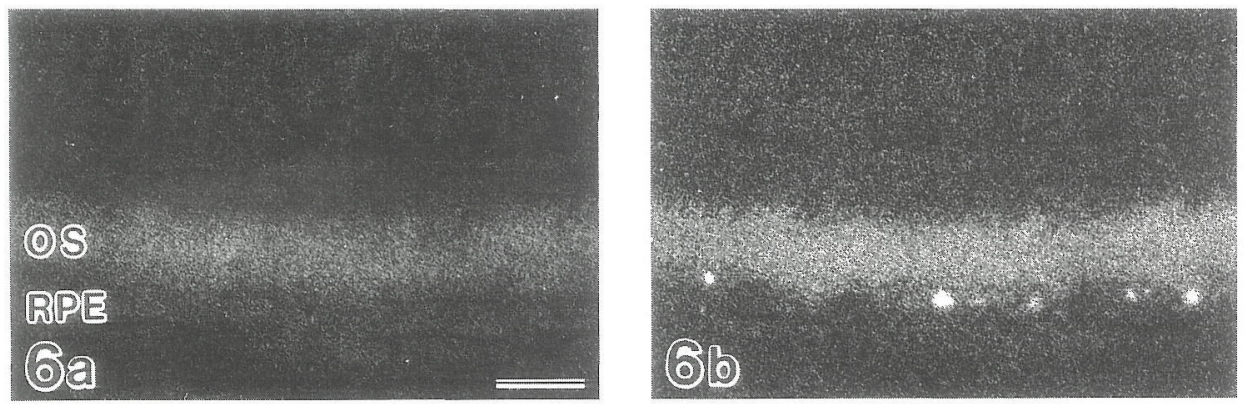

FIgs. 6a, b. Monochromatic CL images of a hypervitaminosis A rat retina at $325 \mathrm{~nm}$ (a) and $445 \mathrm{~nm}$ (b). The granular CL of the RPE consists of the CL peak at $445 \mathrm{~nm}$. Bar $=20 \mu \mathrm{m}$.
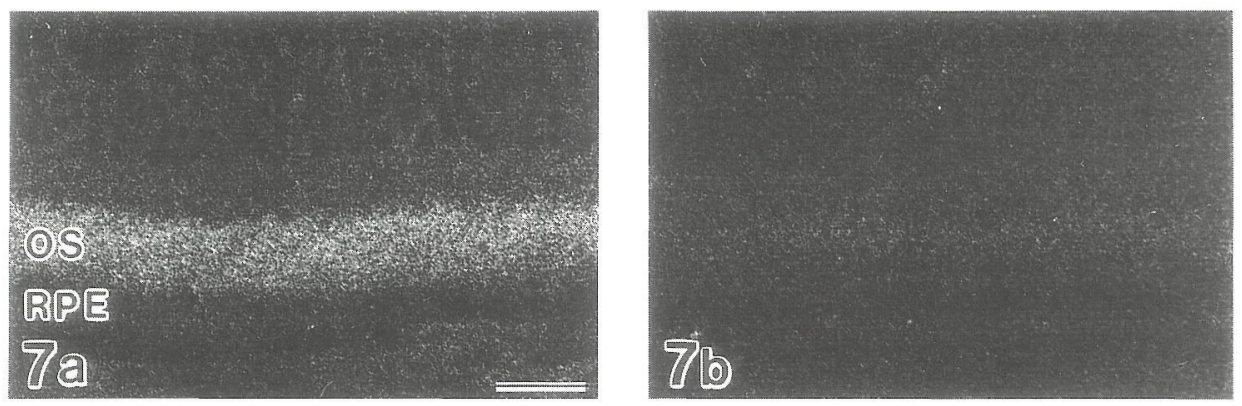

Figs. 7a, b. Monochromatic CL images of a hypovitaminosis A rat retina at $325 \mathrm{~nm}$ (a) and $445 \mathrm{~nm}$ (b). The GL of $445 \mathrm{~nm}$ is very weak and the fluorescent granules of the RPE is found to severely decrease or disappear. Bar $=20 \mu \mathrm{m}$. 

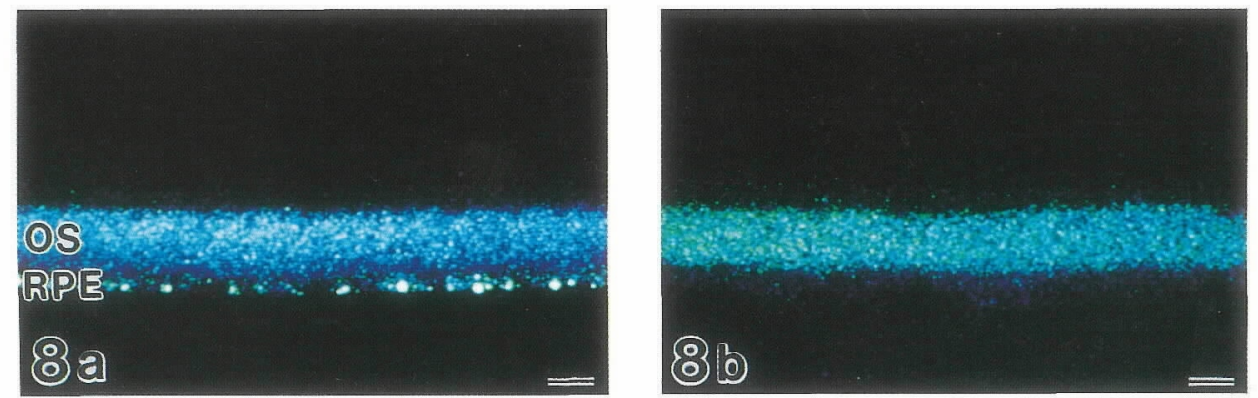

FIGs. 8a, b. Color CL images of the light- (a) and dark- (b) adapted rat retinas. The number of fluorescent granules in the RPE is apparently increased in the light-adapted retina. Bars $=20 \mu \mathrm{m}$.
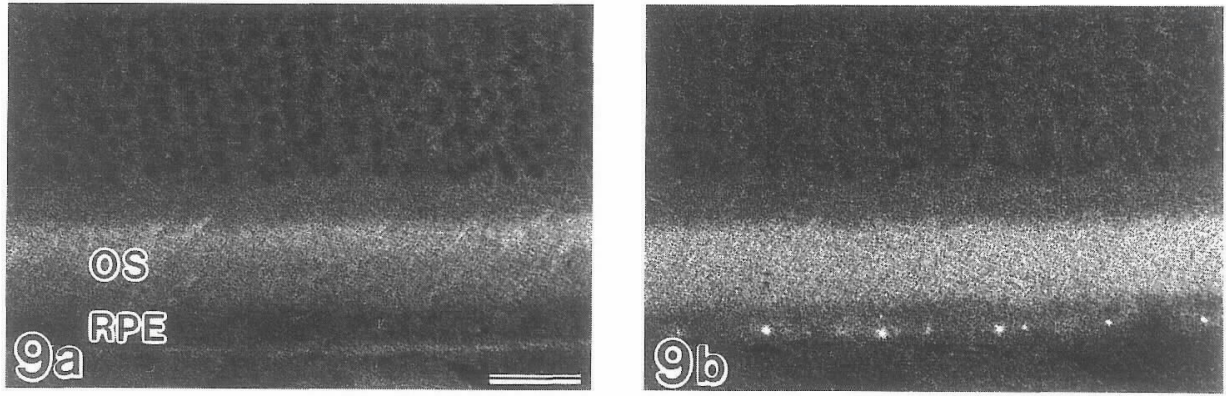

Figs. 9a, b. Monochromatic CL images of a light-adapted rat retina at $325 \mathrm{~nm}$ (a) and $445 \mathrm{~nm}$ (b). The granular CL of $445 \mathrm{~nm}$ consists of the CL peak at $445 \mathrm{~nm}$. Bar $=20 \mu \mathrm{m}$.
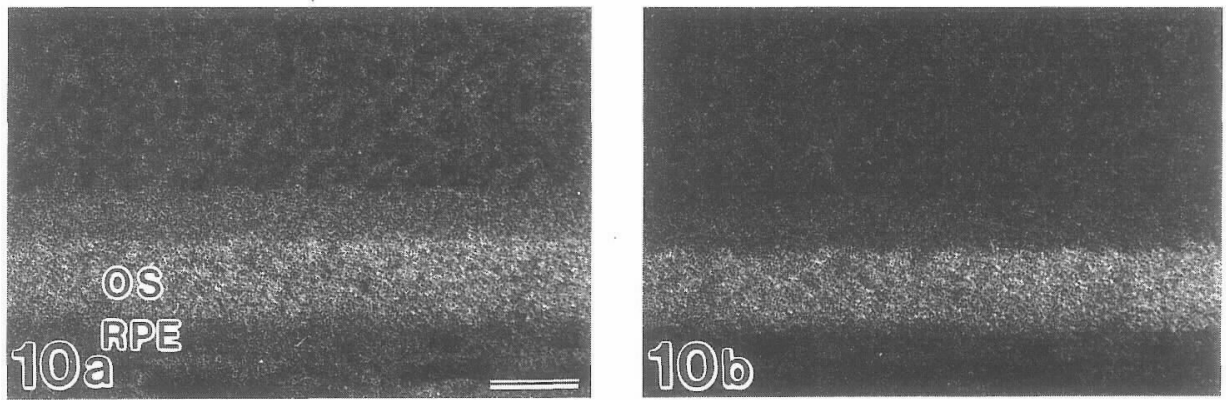

FIGs. 10a, b. Monochromatic CL images of a dark-adapted rat retina at $325 \mathrm{~nm}$ (a) and $445 \mathrm{~nm}$ (b). The $445 \mathrm{~nm}$ CL is weaker than that of the light-adapted rat. Bar $=20 \mu \mathrm{m}$. 

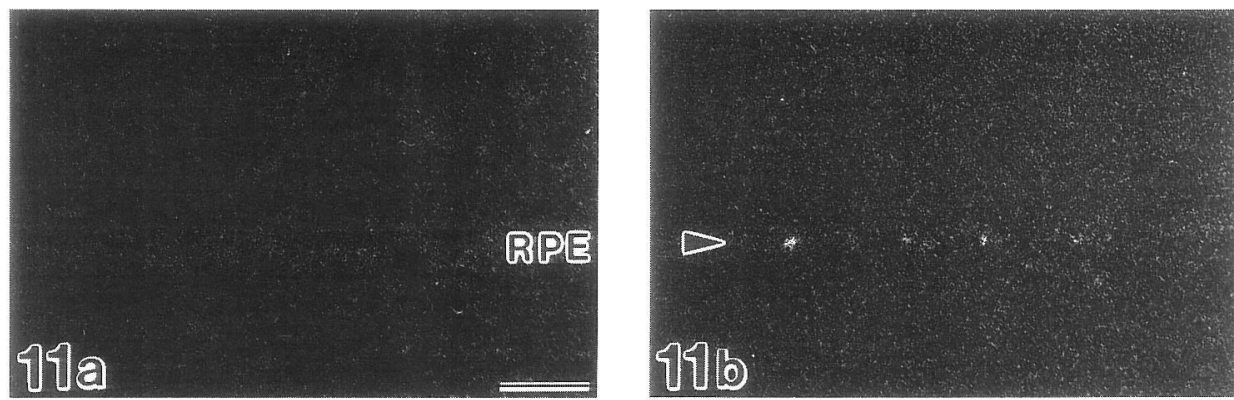

FIGs. 11a, b. Monochromatic CL images of a new born rat retina at $325 \mathrm{~nm}$ (a) and $445 \mathrm{~nm}$ (b). Granular CL of $445 \mathrm{~nm}$ in the RPE (arrowheads) is already found. Bar $=20 \mu \mathrm{m}$.
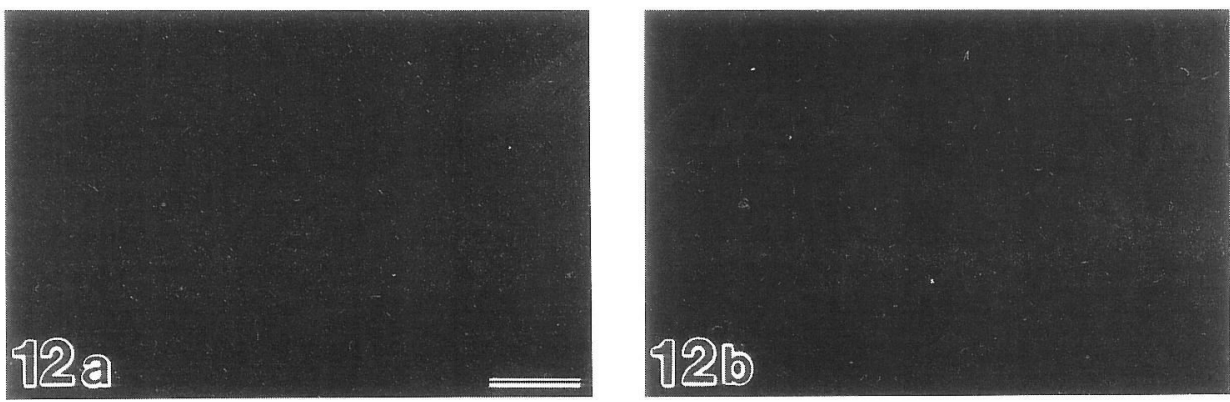

FIGS. 12a, b. Monochromatic CL images of a rat retina on the 12 th day after birth at $325 \mathrm{~nm}$ (a) and $445 \mathrm{~nm}$ (b). The CL in the RPE is found almost disappeared. Bar $=20 \mu \mathrm{m}$.
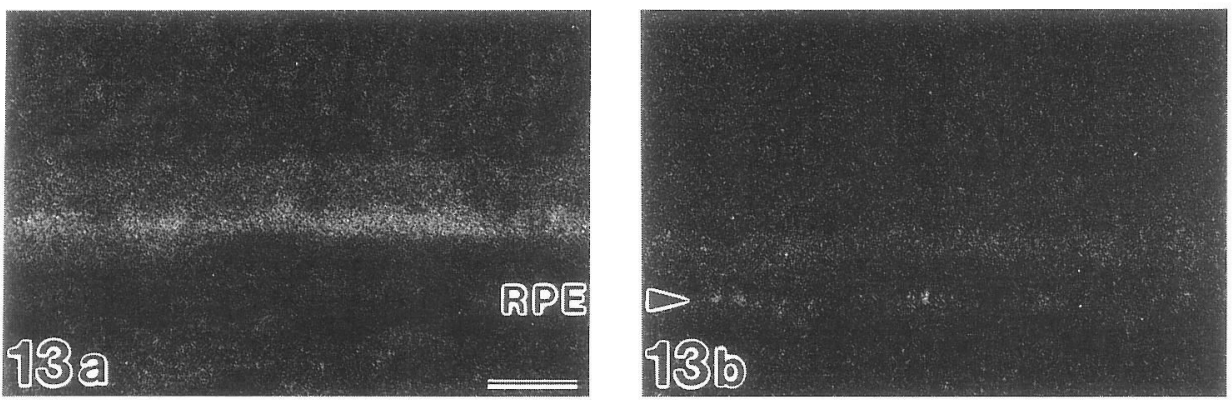

Figs. 13a, b. Monochromatic CL images of a rat retina on the 13 th day after birth at $325 \mathrm{~nm}$ (a) and $445 \mathrm{~nm}$ (b). Weak band-like CL of $325 \mathrm{~nm}$ and $445 \mathrm{~nm}$ is found just anterior to the RPE. The granular CL of $445 \mathrm{~nm}$ in the RPE is found to increase again (arrowhead). Bar $=20 \mu \mathrm{m}$. 

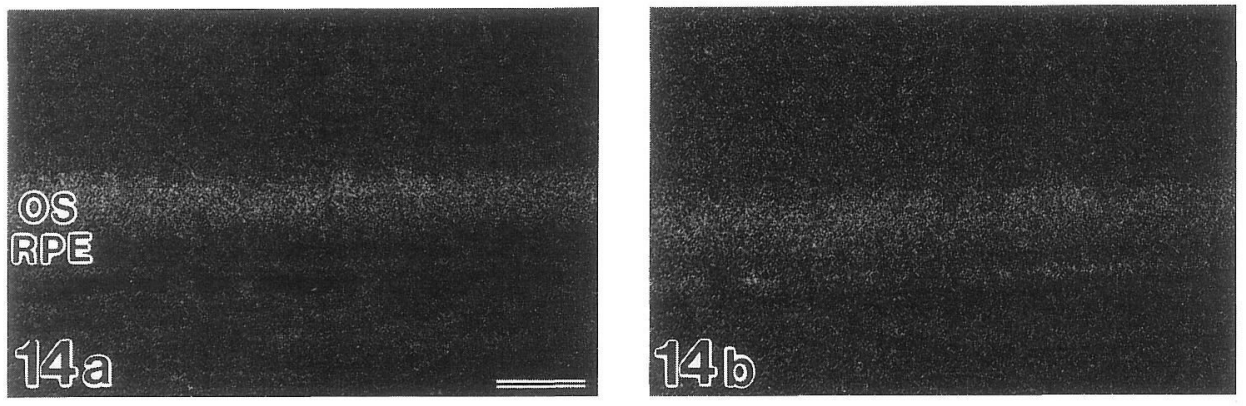

FIGs. 14a, b. Monochromatic CL images of a rat retina on the 14 th day after birth at $325 \mathrm{~nm}$ (a) and $445 \mathrm{~nm}$ (b). The band-like CL is found in both $325 \mathrm{~nm}$ and $445 \mathrm{~nm}$. Bar $=20 \mu \mathrm{m}$.
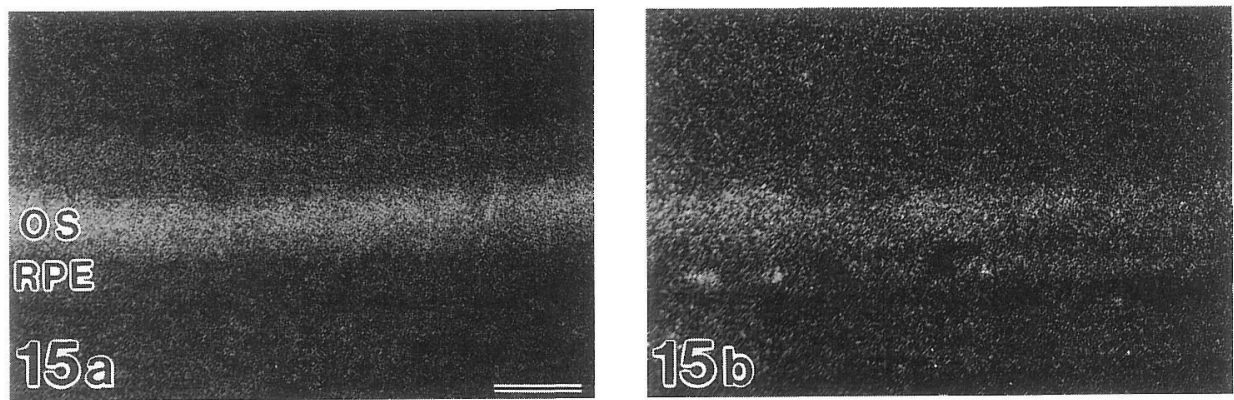

FIGs. 15a, b. Monochromatic CL images of a rat retina at the end of three weeks after birth at $325 \mathrm{~nm}$ (a) and $445 \mathrm{~nm}(\mathrm{~b})$. The CL pattern is similar to that of adult rats. $B a r=20 \mu \mathrm{m}$.

ed retinas, although a little decrease in CL intensity was found in the latter (data not shown).

To confirm the hypovitaminosis A in vitamin A-deficient dieted rats in experiment 1 , unfixed rat livers of this group were examined by the ACFEM according to the method of Itoh et al. (19), and the animals which showed no CL emission from liver were used for the experiments.

1. Hyper- and hypovitaminosis A

Band-like and granular CL images in strong blue-green color were observed from the hypervitaminosis A rat retinas (Fig. 3a). Comparison of the secondary electron images with the color CL images in higher magnification revealed that the band-like CL was emitted from the outer segment (OS) and the granular CL from lipid droplets of the retinal pigment epithelial cells (RPE) (Figs. 3b, c). By CL spectrometry, CL spectra with two peaks at about $325 \mathrm{~nm}$ and $445 \mathrm{~nm}$ were obtained (Fig. 4).

In the retina of the hypovitaminosis A rat, blue-green CL which also had the same two wavelength peaks was observed (Fig. 5a), while the GL intensity was apparently lower than that of the hypervitaminosis $\mathrm{A}$ rats when observed under the same accelerating voltage and electron beam current. Especially, the granular CL in the RPE decreased both in number and intensity. Secondary electron images of the 
vitamin A-deficient retinas showed severe destruction of the plasma membrane of the OS (Fig. 5b).

In order to elucidate the distribution pattern of the two spectral components of the CL in the hyper- and hypovitaminosis A rat retinas, monochromatic CL images at each peak wavelength were observed by connecting a PMT next to the monochrometer. The monochromatic CL images revealed that the granular CL of the RPE, which apparently decreased in the hypovitaminosis A rat retinas, consisted of the CL peak of $445 \mathrm{~nm}$ only and did not contain the other CL peak components (Figs. 6, 7). Relatively weak CL of $445 \mathrm{~nm}$ was also found diffusely distributed in the OS (Fig. 6b). In the hypovitaminosis A rat retina, the CL of $445 \mathrm{~nm}$ in both the OS and the RPE was very weak or hardly detected (Fig. 7b). The GL of $325 \mathrm{~nm}$ was found predominantly in the OS similarly in both the hyper- and hypovitaminosis A rat retinas (Figs. 6a, 7a).

2. light and dark adaptation

The CL spectra of the light- and dark-adapted rat retinas showed the same peak wavelengths as those of the hyper- and hypovitaminosis A rats (data not shown). Comparing the two color images of light- and dark-adapted rat retinas, the granular CL of the RPE was observed to increase after light adaptation (Figs. 8a, b). The monochromatic CL images of each peak wavelength showed that the granular CL of the RPE consisted of the peak at $445 \mathrm{~nm}$. The CL of $445 \mathrm{~nm}$ in both the OS and RPE was apparently weak in the dark-adapted eye, but the band-like CL of $325 \mathrm{~nm}$ in the OS did not change (Figs. 9, 10).

3. postnatal development

The CL spectra of infant rat retinas also showed the same wavelength peaks as those of adults although the intensity was low up to three weeks after birth (data not shown). In the new born rat retinas, faint blue-green granular CL was found in the RPE layer. Monochromatic CL images at each peak wavelength revealed that this granular CL consisted of the peak at $445 \mathrm{~nm}$ (Fig. 11). The granular CL in the RPE gradually decreased up to 12th day after birth (Fig. 12). On the 13th day, band-like CL of $325 \mathrm{~nm}$ and faint CL of $445 \mathrm{~nm}$ first appeared just anterior to the RPE (possibly derived from immature OS) and the granular CL of $445 \mathrm{~nm}$ in the RPE was found to increase again (Fig. 13). On the 14th day, the band-like CL was found brighter in $325 \mathrm{~nm}$ and also in $445 \mathrm{~nm}$, and its width was larger than that of the previous day (Fig. 14). At the end of the 3rd week, the CL pattern of the retina seemed to be similar to that of adult rats (Fig. 15).

\section{DISCUSSION}

CL of biomolecules is derived from loosely bound $\pi$-electrons and so gives us information about chemical bonds (9). This characteristic of CL suggests a possibility for CL analysis technique as a new analytical device in biology. However, CL from biological specimens is generally a weak luminescence signal and easily fades under a high electron beam current or repeated electron beam scanning (10): this is called the "beam effect", and has made it difficult to utilize CL in biology. Therefore, a system which can effectively collect CL and minimize the beam effect has been required.

In the early days of CL research, the CL collection system consisted of a light pipe or lens and PMT $(8,32)$, and so did our system in its early development. In 
order to achieve a large solid angle of CL emission, various-shaped concave mirrors such as a parabolic $(3,24)$ and a hemispheric mirror $(20)$ were introduced. An ellipsoidal mirror was first adopted in 1972 by Hörl (14), and we also adopted this type of mirror to maintain a large solid angle and keep transmission losses of CL as low as possible.

We have developed a color CL-imaging technique using a triple ellipsoidal mirror which was designed to divide CL into three primary color components. By reconstructing the three color components into a single color CL image using a memory system, we succeeded in obtaining a color CL image at an organelle level. A color CL image can be a map showing the localization of CL-emitting substances at a glance and can be applied to labelling techniques in cytochemistry.

In order to minimize the beam effect and also to preserve the ultrastructure, we adopted a modification of the simple cryo-SEM method (18) and a cryo-holder. Hörl and Roschger (15) described no essential differences between the CL properties of specimens between $4.2 \mathrm{~K}$ (temperature of liquid helium) and $77 \mathrm{~K}$ (temperature of liquid nitrogen), but at room temperature the CL properties were completely changed. Therefore, we adopted a cryo-holder cooled by liquid nitrogen, which is low-cost and easy to operate compared to that by liquid helium. The other way to minimize the beam effect is to reduce the total amount of the electron irradiation. In the early steps of development of the ACFEM, we introduced a single-photon counting technique for CL spectrometry to detect faint CL under very low electron beam current. However, during a series of experiments, it was found that the CL of biological specimens especially in the ultraviolet region is quenched within several seconds; on the other hand, it took several minutes to scan a wide range of wavelengths, such as 300 to $700 \mathrm{~nm}$, by the monochrometer and stepping motorized scanning controller which we were using. In order to reduce the time needed for CL spectrometry, an SMA system was introduced. By using this system, the measuring time of the CL spectra was significantly reduced to a millisecond order. The SMA combined with a gate pulse generator can be used to measure the CL lifetime using a short-excitation pulse of the electron beam. By employing time-resolved CL spectroscopy, identification of biomolecules by means of the CL technique will be a more powerful tool in the biological field in the future.

In a previous study, Itoh et al. (19) reported that vitamin A esters stored in hypervitaminosis A rat liver could be detected by CL analysis. It is well known that vitamin A serves as a source of chromophore for rhodopsin and changes its distribution and chemical form during a visual cycle. In this study, we first observed the CL of adult rat retinas in different conditions in experiments 1 and 2: hyper- and hypovitaminosis $\mathrm{A}$, and light and dark adaptation, respectively. The changes of content and distribution of vitamin $\mathrm{A}$ and its esters in rat retinas under these conditions have been extensively studied histo- and biochemically by many investigators $(1,13$, $21,23,27,31,34,36)$. Therefore the CL observation under these conditions seemed to be a good model to evaluate the applicability of the ACFEM to retinal research. Our findings in experiments 1 and 2 are summarized as follows: 1) Rat retinas revealed two patterns of CL emission: band-like and granular CL, which corresponded to the OS and RPE, respectively. 2) The CL from rat retina has two spectral peaks at around 325 and $445 \mathrm{~nm}$. The granular CL of the RPE consists only of the CL of the latter peak, and the CL of the OS has both peaks. 3) In the hypovitaminosis A rat 
retinas, the CL of $445 \mathrm{~nm}$ in both the OS and the RPE was weak or hardly detected, while that of the hypervitaminosis A rats was intense. 4) In the dark-adapted rat retinas, the CL of $445 \mathrm{~nm}$ in both the OS and the RPE was weak compared to that of the light-adapted eyes.

It has been reported that excess vitamin $\mathrm{A}$ is stored in the ester form in the lipid droplets of the RPE $(5,11,23)$. Krinsky (23) reported that about $90 \%$ of total vitamin $A$ in the bovine RPE is esterified in light-adapted eyes. Our results of hyperand hypovitaminosis A rat retinas showed that the granular CL of the lipid droplets in the RPE increased in both number and intensity in hypervitaminosis A while that in hypovitaminosis A was found to be few in number and weak. Therefore, it is strongly suggested that the granular CL of $445 \mathrm{~nm}$ in the RPE is derived from vitamin A esters. On the other hand, the origin of the band-like CL in the OS seems more complicated. During the visual cycle, several conformational changes of retinoids take place. During dark adaptation, hydrolysis of retinyl esters, diffusion of retinol into the OS, oxidation to retinal and its isomerization are known to occur, and so do the reverse reactions during light adaptation (11, 16, 23, 36). Zimmerman (36) reported the distribution and proportion of vitamin A compounds during the visual cycle in the rat using radioisotopes and a thin layer chromatography technique. According to his results, the amount of retinyl ester both in the retina and the RPE significantly increased after 60 min of light adaptation, while the amounts of retinal and retinol rapidly decreased. On the contrary, in the dark-adapted eye, the amount of retinyl esters in the retina and RPE decreased to about one-third and one-seventh, respectively, while retinal showed a significant increase. In our observation, the CL of $445 \mathrm{~nm}$ in the OS slightly decreased after dark adaptation and that in the RPE was apparently diminished. These results support the idea that the CL of $445 \mathrm{~nm}$ in the OS is also derived mainly from retinyl esters as in the RPE. The CL of $325 \mathrm{~nm}$ in the OS was found in all the conditions examined. The predominant localization of this CL peak in the OS suggests its relationship with opsin (25), the binding protein of the chromophore.

Morphological study on retinal development has been done for several decades $(12,28,35)$. Weidman and Kuwabara (35) have reported a precise morphological development of postnatal rat retina; the OS began developing during the 5 th postnatal day and were well formed at the end of two weeks, and the RPE was already found in new born rats as a single cell layer adjacent to undifferentiated neuroblastic cells. In experiment 3 , we observed the CL of postnatal developing rat retinas. The granular CL of $445 \mathrm{~nm}$ in the RPE was already found in new born rats. This finding indicates that the RPE is functioning as a vitamin A storing cell already at the time of birth. The decrease of the CL in the RPE up to 12th day suggests that vitamin A stored during the fetal period is consumed during this period. The band-like CL of $445 \mathrm{~nm}$ in the OS was first found on the 13th postnatal day, and became brighter on the following day. The electroretinogram (ERG) of the developing rat retina was recordable on the 13th day and became the same as that of adult rats on the 14th day (35); it was speculated that this ERG response is related to the formation of synapses in the inner plexiform layer. Our results also suggest that the OS begins to function on the 13th postnatal day in vitamin A turnover, although the morphological development of the OS begins earlier $(12,35)$.

Greenberg and Popper (13) demonstrated the distribution of vitamin A in rat retina using a conventional fluorescence microscope. However, they could not detect 
the difference in the distribution of vitamin A between hyper- and hypovitaminosis $\mathrm{A}$ rats because of difficulties in differentiating vitamin A fluorescence from other autofluorescence by light microscopy. Other conventional histochemical techniques for the detection of vitamin $\mathrm{A}$ at light microscopic level have several weak points as described by Amemiya (1); the antimony trichloride method cannot act upon vitamin A in the presence of water, the method using conc. sulphuric acid severely corrodes tissues, and the method of iodine-potassium iodide solution can react with lecithin. Vigh-Teichman et al. (34) demonstrated the immunocytochemical localization of vitamin $\mathrm{A}$ in frog retina using antibodies raised against retinoic acid. This immunocytochemical technique can be used in electron microscopy, but the antibody did not crossreact with retinyl esters.

The results of the present study show that the CL imaging technique using the ACFEM is not only simple and easy but also sensitive enough to detect the distribution of vitamin A esters in the eye in both physiological and pathological conditions.

\section{ACKNOWLEDGMENTS}

We are grateful to Ms. K. Okano and Mr. K. Ozaki for their technical assistance. We also wish to thank Mr. S. Atkins for improving the English of this manuscript.

\section{REFERENCES}

1. Amemiya, T.: Histochemical examination of retinas of vitamin A. Report 1. Distribution of vitamin A in the retina. Acta Soc. Ophthalmol. Jap. 71; 2079-2083, 1967.

2. Basu, S.: Cathodoluminescence studies: from chromatin and chromosome to human hair. In Advances in Microscopy, ed. by R. R. Cowden and F. W. Harrison, Alan R. Liss Inc., New York, 1983, p. 265-268.

3. Bond, E. F., Beresford, D. and Haggis, G. H.: Improved cathodoluminescence microscopy. J. Microsc. 100; 271-282, 1974.

4. Boyde, A. and Reid, S. A.: New methods for cathodoluminescence in the scanning electron microscope. Scan. Electron Microsc. IV; 1803-1814, 1983.

5. Bridges, C. D. B.: Storage distribution and utilization of vitamin A in the eyes of adult amphibians and their tadpoles. Vision Res. 15; 1311-1323, 1975.

6. Bröcker, W. and Pfefferkorn, G.: Applications of the cathodoluminescence method in biology and medicine. Scan. Electron Microsc. II; 125-132, 1979.

7. Cavellier, J. F. and Berry, J. P.: Cathodoluminescence applied to the study of human glomerulonephritis. Phys. Med. Biol. 30; 825-830, 1985.

8. Cavellier, J. F., Berry, J. P. and Lagrue, G.: Cathodoluminescence applied to immunofluorescence: present state and improved technical prospects by prism spectrometer light selection. Histochemistry 57; 313-322, 1978.

9. De Mets, M.: Cathodoluminescence of organic chemicals. In Principles and Techniques of Scanning electron Microscopy, vol. 2, ed. by M. A. Hayat, Van Nostrand Reinhold, New York, 1974, p. 1-20.

10. De Mets, M. and Lagasse, A.: An investigation of some organic chemicals as cathodoluminescent dyes using scanning electron microscope. J. Microsc. 94; 151-156, 1971.

11. Dowling, J. E.: Chemistry of visual adaptation in the rat. Nature 188; 114-118, 1960.

12. Galbavy, E. S. J. and Olson, M. D.: Morphogenesis of rod cells in the retina of albino rat: a scanning electron microscopic study. Anat. Rec. 195; 707-718, 1979. 
13. Greenberg, R. and Popper, H.: Demonstration of vitamin A in the retina by fluorescence microscopy. Am. J. Physiol. 134; 114-118, 1941.

14. Hörl, E. M.: Scanning electron microscopy of biological material using cathodoluminescence. Micron 3; 540-545, 1972.

15. Hörl, E. M. and Roschger, P.: CL SEM investigations of biological material at liquid helium and liquid nitrogen temperatures. Scan. Electron Microsc. I; 285-292, 1980.

16. Hubbard, R. and Colman, A. D.: Vitamin A content of the frog eye during light and dark adaptation. Science 130; 977-978, 1959.

17. Hurter, T., Bröcker, W. and Hossmann, K. A.: Evaluation of vasogenic edema in experimental brain tumors by cathodoluminescence and fluorescence microscopy. Histochemistry 72; 249254, 1981.

18. Inoue, T., Koike, H. and Sato, T.: Direct SEM observation of non-fixed, rapidly frozen intracellular structures. J. Electron Microsc. 32, 275, 1983.

19. Itoh, T., Fujimoto, T., Koike, H., Inoue, T. and Ogawa, K.: Color cathodoluminescence images and cathodoluminescence spectra analysis of biological materials. Acta histochem. cytochem. 19; 621-633, 1986.

20. Judge, F. J., Stubbs, J. M. and Philip, J.: A concave mirror, light pipe, photon collecting system for cathodoluminescent studies on biological specimens in the JSM2 scanning electron microscope. J. Phys. (E) 7; 173-174, 1974.

21. Katz, M. L., Drea, C. M. and Robinson, W. G. Jr.: Dietary vitamin A and E influence retinyl ester composition and content of the retinal pigment epithelium. Biochem. Biophys. Acta 924; 432-441, 1987.

22. Koike, H., Nakano, T., Fujimoto, T. and Ogawa, K.: Analytical color fluorescence electron microscope. In EUREM 88, vol. 3: Biology, ed. by H. G. Dickinson and P. J. Goodhew, Institute of Physics, Bristol and Philadelphia, 1988, p. 591-592.

23. Krinsky, N. I.: The enzymatic esterification of vitamin A. J. Biol. Chem. 232; 881-894, 1958.

24. Muir, M. D., Grant, P. R., Hubbard, G. and Mundell, J.: Cathodoluminescence spectra. Scan. Electron Microsc. I; 401-408, 1971.

25. Nir, I., Cohen, D. and Papermaster, D. S.: Immunocytochemical localization of opsin in the cell membrane of developing rat retinal photoreceptors. J. Cell Biol. 98; 1788-1795, 1984.

26. Nishimura, K., Nakano, T., Koike, H., Tomimori, H., Kawarada, H., Hiraki, A. and Ogawa, K.: Observing diamond defects with an analytical color fluorescence electron microscope. In Optical and Optoelectronic Applied Science and Engineering, Proc. SPIE, 1990. (in press).

27. Noell, W. K., Delmelle, M. G. and Albrecht, R.: Vitamin A deficiency effect on retina: dependence on light. Science 172; 72-76, 1971.

28. Ordy, J. M., Samorajski, T., Collins, R. L. and Cleveland, M. S.: Postnatal development of vision in a subhuman primate (Macaca Mulatta)-A multidisciplinary study. Arch. Ophthalmol. 73; 674-686, 1965.

29. Pearce, R. F. W. and Hays, T. L.: Scanning electron microscopy of biological materials. Nature 210; 1049, 1966.

30. Pfefferkorn, G., Bröcker, W. and Hastenrath, M.: The cathodoluminescence method in the scanning electron microscope. Scan. Electron Microsc. I; 251-258, 1980.

31. Robinson, W. G. Jr. and Kuwabara, T.: Vitamin A storage and peroxisomes in retinal pigment epithelium and liver. Invest. Ophthalmol. Vis. Sci. 16; 1110-1117, 1977.

32. Schmidt, E. H., Bröcker, W., Wagner, H., Pfefferkorn, G. and Beller, F. K.: Glomerular microcapillary thrombosis demonstrated by the new technique of immunocathodoluminescence. Am. J. Pathol. 81; 43-46, 1975.

33. Soni, S. L., Kalnis, V. I. and Haggis, G. H.: Localization of caps on mouse B lymphocytes by scanning electron microscopy. Nature 255; 717-719, 1975.

34. Vigh-Teichmann, I., Vigh, B., Szél, A., Röhlich, P. and Wirtz, G. H.: Immunocytochemical localization of vitamin $A$ in the retina and pineal organ of the frog, Rana esculenta. Histochemistry 88; 533-543, 1988. 
35. Weidman, T. and Kuwabara, T.: Postnatal development of the rat retina-An electron microscopic study. Arch. Ophthalmol. 79; 470-484, 1968.

36. Zimmerman, W. F.: The distribution and proportions of vitamin A compounds during the visual cycle in the rat. Vision Res. 14; 795-802, 1974. 